\title{
Latitudinal characteristics of GPS derived ionospheric TEC: a comparative study with IRI 2012 model
}

\author{
Monti Chakraborty ${ }^{1}$, Sanjay Kumar ${ }^{2}$, Barin Kumar De ${ }^{1}$, Anirban Guha ${ }^{1}{ }^{\star}$ \\ ${ }^{1}$ Tripura University, Department of Physics, Suryamaninagar, Tripura, India \\ ${ }^{2}$ Nanyang Technological University (NTU), School of Electrical and Electronic Engineering (EEE), Singapore
}

\author{
Article history \\ Received November 9, 2013; accepted August 28, 2014. \\ Subject classification: \\ Ionosphere, GPS, Ionospheric total electron contents, IRI model.
}

\begin{abstract}
The present study investigates the variation of Total Electron Content (TEC) using Global Positioning System (GPS) satellites from four equatorial to mid-latitudes stations over a period of one year. The stations are Port Blair $\left(11.63^{\circ} \mathrm{N}, 92.70^{\circ} \mathrm{E}\right)$, Agartala $\left(23.75^{\circ} \mathrm{N}, 91.25^{\circ} \mathrm{E}\right)$, Lhasa $\left(29.65^{\circ} \mathrm{N}, 91.10^{\circ} \mathrm{E}\right)$ and Urumqi $\left(43.46^{\circ} \mathrm{N}, 87.16^{\circ} \mathrm{E}\right)$. The diurnal, monthly and seasonal variations of TEC have been explored to study its latitudinal characteristics. Analysis of TEC data from these stations reveals the characteristics of latitudinal variation of Equatorial Ionospheric Anomaly (EIA). To validate the latest IRI 2012 model, the monthly and seasonal variations of GPS-TEC at all the four stations have been compared with the model for three different topside options of electron density, namely, NeQuick, IRI-01-corr and IRI-2001. TEC predictions from IRI2001 top side electron density option using IRI 2012 model overestimates the observed TEC especially at the low latitudes. TEC from IRI- NeQuick and IRI-01-corr options shows a tendency to underestimate the observed TEC during the day time particularly in low latitude region in the high solar activity period. The agreement between the model and observed values are reasonable in mid latitude regions. However, a discrepancy between IRI 2012 derived TEC with the ground based observations at low latitude regions is found. The discrepancy appears to be higher in lowlatitude regions in comparison to mid latitude regions. It is concluded that largest discrepancy in TEC occur as a result of poor estimation of the $\mathrm{hmF} 2$ and foF2 from the coefficients.
\end{abstract}

\section{Introduction}

The temporal and spatial variation of Total Electron Contents (TEC) at the equatorial and low latitude regions are significant compared to mid latitude regions. This dynamic nature is due to various processes like equatorial ionization anomaly (EIA), Equatorial Electrojet (EEJ), and equatorial spread-F (ESF) irregularities etc. Associated with it, is also challenging task to model it with relevant accuracy [Sridharan et al. 1994, Kumar and Singh 2009, Aggarwal 2011]. TEC is an im- portant ionospheric parameter which is defined as the total number of electrons present within an area of $1 \mathrm{~m}^{2}$ cross section along the integrated path from the satellite to the receiver. GPS receiver is one of the viable tools for TEC study. GPS signals traverse the ionosphere carrying the signatures of the dynamic medium and thus offer an excellent opportunity for ionospheric research. The TEC study is important as this parameter helps to reveal the variability of ionospheric behavior during the solar quiet and disturbed days and thus has significant practical applications in satellite navigation, time delay and range error corrections for single frequency GPS satellite signal receivers.

The ionosphere being a complex physical system, exhibit different features like diurnal, seasonal and spatial variability. The seasonal variation of TEC in equatorial and low latitude regions depend on various factors such as solar zenith angle variation, thermospheric neutral composition changes, change in direction of neutral wind and equatorial electroget (EEJ) strength and studied by a number of workers [Rastogi and Sharma 1971, da Rosa et al. 1973, Balan et al. 1986, 1991, 1997, Warnant 2000, Wu et al. 2008, Bagiya et al. 2009, Kumar et al. 2012]. Due to the unequal heating of the two hemispheres, neutral constituents are transported from the summer (hot) to the winter (cold) hemisphere. As a result, an increase of the $\mathrm{O} / \mathrm{N} 2$ ratio caused by the convection of atomic oxygen is formed in the winter hemisphere as compared to summer hemisphere. Therefore, the recombination in winter hemisphere is weaker than that in the summer hemisphere, which results in the relatively higher electron concentration in winter hemisphere [Rishbeth and Setty 1961, Johnson 1963, Torr and Torr 1973, Balan et al. 1997]. Another possible mechanism for this seasonal anomaly 
is the change of direction of neutral wind. A meridional component of neutral wind blows from the summer to the winter hemisphere which can reduce the crest value during summer solstice as it blows in an opposite direction to the plasma diffusion process originating from the magnetic equator; at the equinoxes, meridional winds from equator blows pole-wards should result in a high ionization crest value. Based on this scenario, a seasonal effect on the crest should be expected with the crest maximum at the equinoxes [Bramley and Young 1968, Stening 1992, Wu et al. 2004, 2008]. The distribution and characteristics of TEC over low, mid and high latitude regions have been investigated by several workers [Lanzerotti et al. 1975, Rama Rao et al. 2006a, Yizengaw et al. 2007, Liu et al. 2008, Bagiya et al. 2009, Jain et al. 2011, Zou et al. 2011, Kumar et al. 2012, Sojka et al. 2012]. The latitudinal variation of the TEC is mostly dictated by the phenomenon of EIA [Appleton 1946, Kumar and Singh 2009, Venkatesh et al. 2011]. So, a systematic study of TEC over different latitudes is very important to get the idea of latitudinal variation and its global distribution.

The in-situ measurements of TEC using the GPS are not possible from all the places on the Earth. So, to understand the global distribution of TEC, some modeled data are essential. For TEC measurements, different empirical models like IRI (International Reference Ionosphere) [Bilitza 2001, Bilitza and Reinisch 2008], SLIM (Semi-Empirical Low-Latitude Ionospheric Model] [Anderson et al. 1987], PRISM (parameterized, real-time ionospheric specification model) [Daniell and Brown 1995], and NeQuick [Nava et al. 2008] are widely used by various workers. Out of these models, IRI stands out to be very popular which is constantly being improved and updated by a scientific committee. Presently, the most recent version of this model is IRI 2012.

The IRI project was jointly initiated by the Committee on Space Research (COSPAR) and by the International Union of Radio Science (URSI) [Rawer et al. 1978, Bilitza 2001, Bilitza and Reinisch 2008]. The worldwide network of ionosondes, the powerful incoherent scatter radars (Jicamarca, Arecibo, Millstone Hill, Malvern, St. Santin), the ISIS and Alouette topside sounders, in-situ instruments on several satellites and rockets are the major sources of data for this model program [Aggarwal 2011]. The IRI model uses two different computer programs as sub-routine to predict the foF2 and hmF2/M (3000)F2. (1) the model developed by the International Radio Consultative Committee [CCIR 1966] and (2) the model developed by Rush et al. [1989] for the International Union of Radio Science (URSI). The monthly median values of foF2, hmF2 and M (3000) F2 from $\sim 10,000$ ground station-months based world wide ionosonde stations during the year 1954-1958 is the base of the CCIR model. The URSI coefficients are based on around 45,000 station-months ionosonde data. These two models use all the compiled observational data and generate numerical coefficient maps using the application of Legendre and Fourier series. These numerical data maps and coefficients are being updated with input of newer data and a newer version of IRI being released.

Many researchers have performed comparative studies on modeled TEC and observed TEC over different region for the improvement of the performance of the model, as in today's scenario, the model data is very important to study the ionosphere. Depending upon these types of observations, the IRI has been steadily being improved from IRI 78 [Rawer et al. 1978], IRI 85 [Bilitza 1986], IRI 1990 [Bilitza 1990], IRI 2000 [Bilitza 2001], and IRI 2007 [Bilitza and Reinisch 2008] and the latest is IRI 2012. Ezquer et al. [1998] have shown that IRI 95 TEC generally overestimates observed TEC during the daily minimum and underestimates in remaining hours of the day. Bhuyan and Borah [2007] have compared the GPS measured TEC with the IRI 2000 model TEC and found that the model TEC overestimated the observed TEC in low latitude. The IRI 2000 contained a topside formulation based on Booker [1977] approach of Skeleten profile. This approach has the disadvantage that in the upper part of the electron density profile $(800 \mathrm{~km})$, the density becomes nearly constant, thus providing discrepancies between experimental and model TEC values [Mukherjee et al. 2010]. IRI 2007 was released with many enhanced features and showed a good agreement with the observed data in the anomaly crest station [Chauhan and Singh 2010]. As the difference between the observed and model value changes with local time and location, it is necessary to compare the TEC data from both observation and model value from maximum number of stations.

The latest release of IRI is IRI 2012. The model is enriched with many new improvements. In this paper, we have studied the diurnal, monthly and seasonal variations of the GPS-TEC and compared with those derived from the latest IRI 2012 model. We have made the comparison over four different stations - one at low latitude (Port Blair - $11.63^{\circ} \mathrm{N}, 92.70^{\circ} \mathrm{E}$ - Geomagnetic lat. $2.11^{\circ} \mathrm{N}$ ), one anomaly at equatorial crest region (Agartala $-23.75^{\circ} \mathrm{N}, 91.25^{\circ} \mathrm{E}-$ Geomagnetic lat. $14.22^{\circ} \mathrm{N}$ ), one at the edge of anomaly crest region (Lhasa $29.65^{\circ} \mathrm{N}, 91.10^{\circ} \mathrm{E}$ - Geomagnetic lat. $20.09^{\circ} \mathrm{N}$ ) and one at the mid latitude region (Urumqi $-43.46^{\circ} \mathrm{N}, 87.16^{\circ} \mathrm{E}$ - Geomagnetic lat. $34.04^{\circ} \mathrm{N}$ ). These four stations are located along nearly the same geographic longitude $\left(90^{\circ}\right.$ $\pm 3^{\circ} \mathrm{E}$ ) provide an opportunity to study latitudinal 
characteristics of the ionosphere. This type of latitudinal comparison of total electron content from around 90 degree longitude using IRI 2012 model is first time in its field. This type of comparison is most important for the improvement of model data as the acceptance of IRI model is increasing progressively in diverse areas for studying the different ionospheric behavior [Maltseva et al. 2012] and its use in HF communication as a ray tracing application [Azzarone et al. 2012, Settimi et al. 2013]. This type of comparative study might be beneficial to the IRI developers and other empirical modelers. It could also be of interest to the users of ionospheric specification tools, such as GPS providers who currently use the Klobuchar model [Klobuchar 1986] for their ionospheric delay corrections.

\section{Organization of data}

In the present study, the TEC measured from GPS based measurements have been used to validate the IRI 2012 model TEC at four stations. The details about these two TEC data are discussed in the following subsections.

\subsection{TEC from GPS measurements}

A GSV 4004B dual frequency receiver manufactured by GPS Silicon Valley is in operation in the equatorial anomaly crest region over Agartala (AGAR). The receiver is programmed to provide TEC and Scintillation data at 1 minute sampling rate. It provides the slant TEC after the satellite and receiver bias correction. The slant TEC is then converted to vertical TEC (VTEC) by using a suitable mapping function at different ionospheric pierce point (IPP) using thin shell model [Klobuchar 1986] according to Equation (1).

$$
V T E C=\left(S T E C-\left[b_{R}+b_{s}\right]\right) S(E l)
$$

where $b_{R}$ and $b_{s}$ are receivers and satellite biases respectively, El is the elevation angle of the satellite in degrees, $S(E l)$ is the obliquity factor with zenith angle $\chi$ at the ionospheric pierce point (IPP). The obliquity factor $S(E l)$ (or mapping function) is defined as in Equation (2):

$$
S(E l)=\frac{1}{\cos (x)}=\frac{1}{\sqrt[\frac{1}{2}]{\left[1-\left\{\frac{R_{E} \cos (E l)}{R_{E}+h}\right\}^{2}\right.}}
$$

where, $R_{E}$ is the Earth's mean radius in $\mathrm{km}, h$ is the height of the ionospheric Shell (in $\mathrm{km}$ ) above the Earth's surface, $\chi$ is the zenith angle and $E l$ is the elevation angle of satellite in degree. For STEC to VTEC conversion, the shell height $350 \mathrm{~km}$, which is valid for Indian low latitude regions [Rama Rao et al. 2006b] has been used for all the latitudes and produces around 3\% error in VTEC at mid latitude. Apart from this the large electron density gradient present in the equatorial region could also be responsible for the error when STEC converted to VTEC. Since, TEC variation may be affected by multipath, troposcatter and water vapor at low elevation angles, we have thus taken the satellite elevation angle high enough so that it only allow rays with $350 \mathrm{~km}$ pierce points within $\pm 1^{\circ}$ of the receiver location. The latitudes and longitudes of ionospheric pierce points (IPPs) have been calculated from the RINEX observation and navigation message data by using standard coordinate transformation formulae and corrections in satellite orbits [Hofmann-Wellenhof et al. 2001]. In this study the different vertical TEC measurements in the cell around the receiver are averaged to estimate the TEC above the receiver. Around the nearly same longitude of our station, we have collected IGS TEC data for other stations. The selected stations are Lhasa (LHAZ), Port Blair (PBR2), and Urumqi (GUAO). Figure 1 shows the location of these stations. The downloaded data from the IGS website are in compact RINEX format. We have converted this compact format in normal RINEX format using FORTRAN script. The satellite bias is corrected using the differential code bias (DCB) files provided by IGS website. The errors in the computation of satellite and receiver bias mainly effect the STEC measurements and thus propagate to VTEC measurements. The experimental results found the satellite and receiver biases to vary from \pm 6 $\mathrm{ns}(1 \mathrm{~ns}=2.85 \mathrm{TECU})$ with estimation precision of the order of $\pm 0.17 \mathrm{~ns}$ and average value of $\sigma 1.17 \mathrm{~ns}$ [Rao 2007]. The biases for the receiver have been estimated

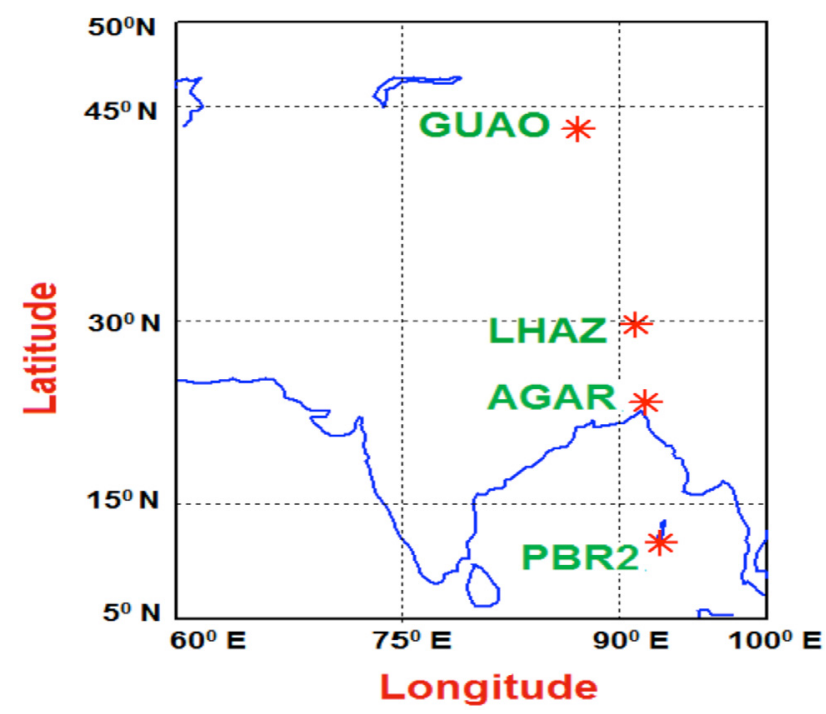

Figure 1. Map showing the geographic location of GPS stations, used in the present study. The geomagnetic latitudes of PBR2, AGAR, LHAZ and GUAM stations respectively are $2.11^{\circ} \mathrm{N}$, $14.22^{\circ} \mathrm{N}, 20.09^{\circ} \mathrm{N}$ and $34.04^{\circ} \mathrm{N}$. 
using Kalman Filter technique [Sardon et al. 1994]. Since Pseudo ranges provide noisy TEC, therefore phase leveling (smoothing) to pseudo range over carrier phase measurements has been made using the approach II discussed by Liu et al. [2005]. As the IRI model predicts the best on the geomagnetic quiet days, we have taken the TEC data for the quiets days only. To get the monthly mean of TEC data we have taken the mean of TEC during international quiet days of each month.

\subsection{TEC from IRI model}

IRI model provides electron density, electron temperature, ion composition, ion temperature and total electron content for any given latitude and longitude, time and date at altitudes ranging from 50 to $2000 \mathrm{~km}$. The IRI model obtains TEC by integrating the electron density profile from the lower boundary to a user-specified upper boundary [Bilitza 2001]. The IRI 2012 TEC consists of bottom side portion (electrons below the F2 peak) and a topside portion (electrons above the F2 peak). The CCIR option is recommended for continental areas, while the URSI option outperforms the CCIR option over the oceanic regions [Aggarwal 2011]. As a result of this update, we have considered TEC data from IRI model using CCIR coefficients only. For the bottom side of the F2 region, the parameters Bill2000 is used in the IRI model which determines the thickness of the bottom side profile. At the present study, we have investigated the discrepancies between experimental and model TEC values in the ionosphere by using NeQuick, IRI-01-corr and IRI-2001 options for topside and by CCIR coefficients of foF2 with IRI 2012.

\section{Results}

In this paper, we have analyzed the GPS data to retrieve total electron content at four stations covering equatorial-EIA to mid-latitude ionosphere during the period from May 2012 to April 2013 near the maximum of solar cycle 24. The model TEC data from IRI model 2012 has also been taken for the same locations and time in to compare with the TEC data obtained from GPS receiver.

\subsection{Diurnal variation of TEC}

Figure 2 shows the typical diurnal variation of TEC from four different stations. TEC variation at all the stations show typical diurnal characteristics such as TEC minimum at predawn and continuing increase with local sunrise attaining a maximum at local noon time followed by a decrease to a minimum during nighttime. As the sun rises, the ionization also increases which causes more concentration of electron near the F2 peak at the ionosphere. Since TEC is directly related with

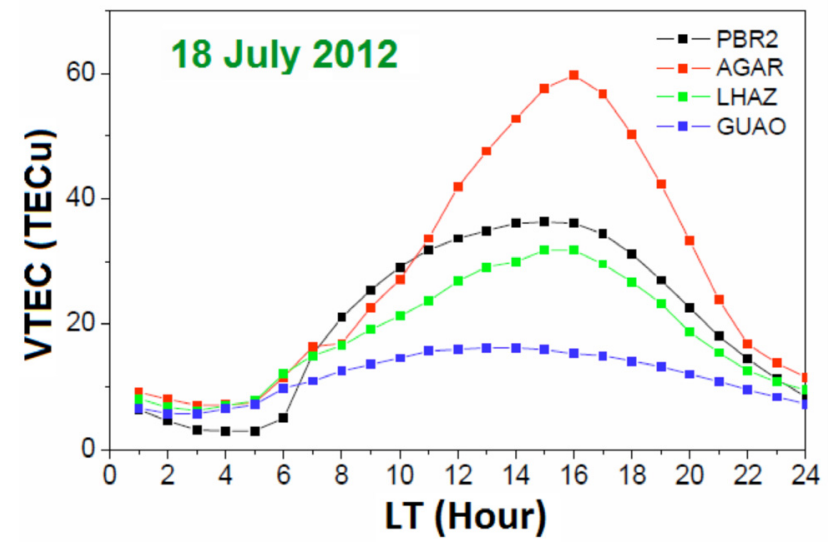

Figure 2. Variation of TEC on a typical day $05 \mathrm{May}-2012$ at four different stations, Port Blair (Geomagnetic lat. $2.11^{\circ} \mathrm{N}$ ), Agartala (Geomagnetic lat. $14.22^{\circ} \mathrm{N}$ ), Lhasa (Geomagnetic lat. $20.09^{\circ} \mathrm{N}$ ) and Urumqi (Geomagnetic lat. $34.04^{\circ} \mathrm{N}$ ).

maximum electron density, it starts to increase and attains maximum value at the local noon time. During daytime, as the temperature increases the loss rate also increases. When the loss rate overcomes the production rate, then gradually TEC starts to decrease.

Among the four stations, the diurnal maximum value of TEC at the crest of the EIA station (AGAR) is highest. Due to enhanced ionization for stronger incident of solar radiation at the equatorial ionosphere, the TEC value is generally largest. As a result of $\mathbf{E} \times \mathbf{B}$ drift in the equatorial region, the electrodynamical lifting of equatorial plasma to $\mathrm{F}$ region heights, the maximum electron density exists in the $\pm 20^{\circ}$ magnetic latitude and this region is known as crest region of EIA [Appleton 1946]. The station AGAR is situated within this EIA crest and therefore in our observation we are getting maximum TEC value for this station. Larger TEC values at the crest region reflect an enhancement of the equatorial anomaly. Comparatively TEC value is less in PBR2, lesser for LHAZ and least for GUAO among the four stations. This variation shows that equatorial and low latitude TEC is higher then the mid latitude TEC. The day time maximum of diurnal variation of TEC is sharp and short-lived within the EIA station AGAR and comparatively wider and long lived day time plateau is present at the equatorial station Port Blair. IRI model TEC also exhibits the similar variation for day time TEC.

\subsection{Seasonal variation of TEC and its comparison with IRI model \\ The seasonal variation of TEC can be influenced by the variation in solar flux over the period of obser- vation [Bhuyan and Borah 2007]. To examine the sea- sonal variations, the observed and modeled TEC are grouped in three seasons: winter (November, Decem- ber, January and February), summer (May, June, July and August), and equinox (March, April, September}


GPS-TEC

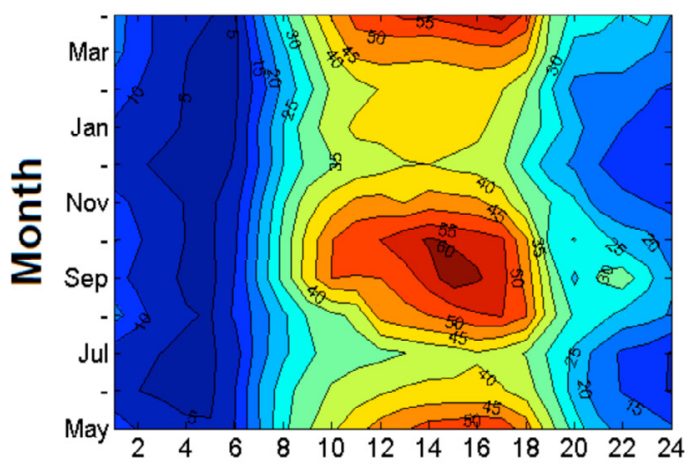

IRI-NeQ

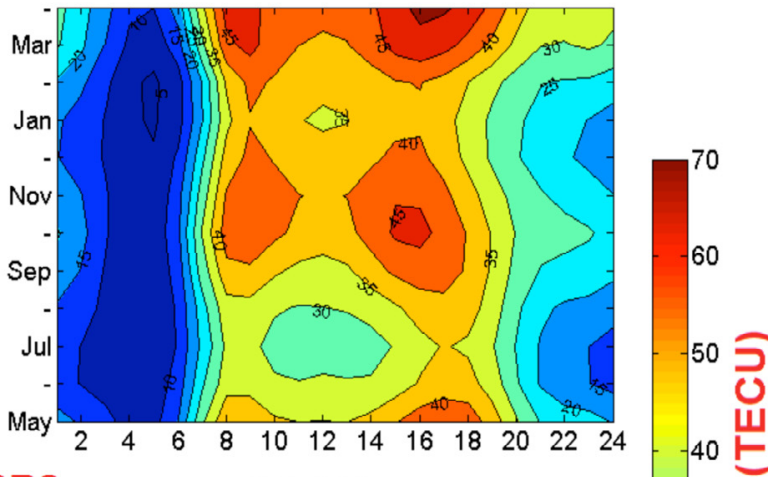

PBR2

IRI-01Corr
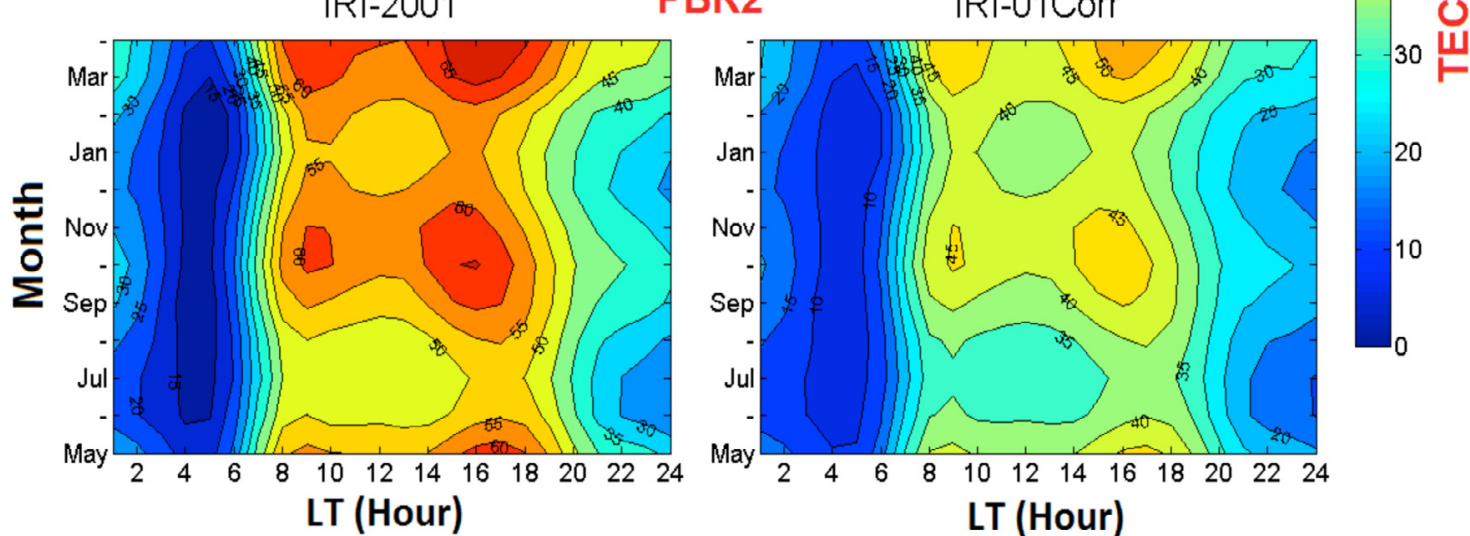

Figure 3. Contour diagram of monthly mean variation of GPS-TEC, IRI 2012 model TEC (NeQuick, IRI- 2001 and IRI-01-corr) at Port Blair (PBR2) (Geomagnetic lat. $2.11^{\circ} \mathrm{N}$ ) from May 2012 to April 2013.
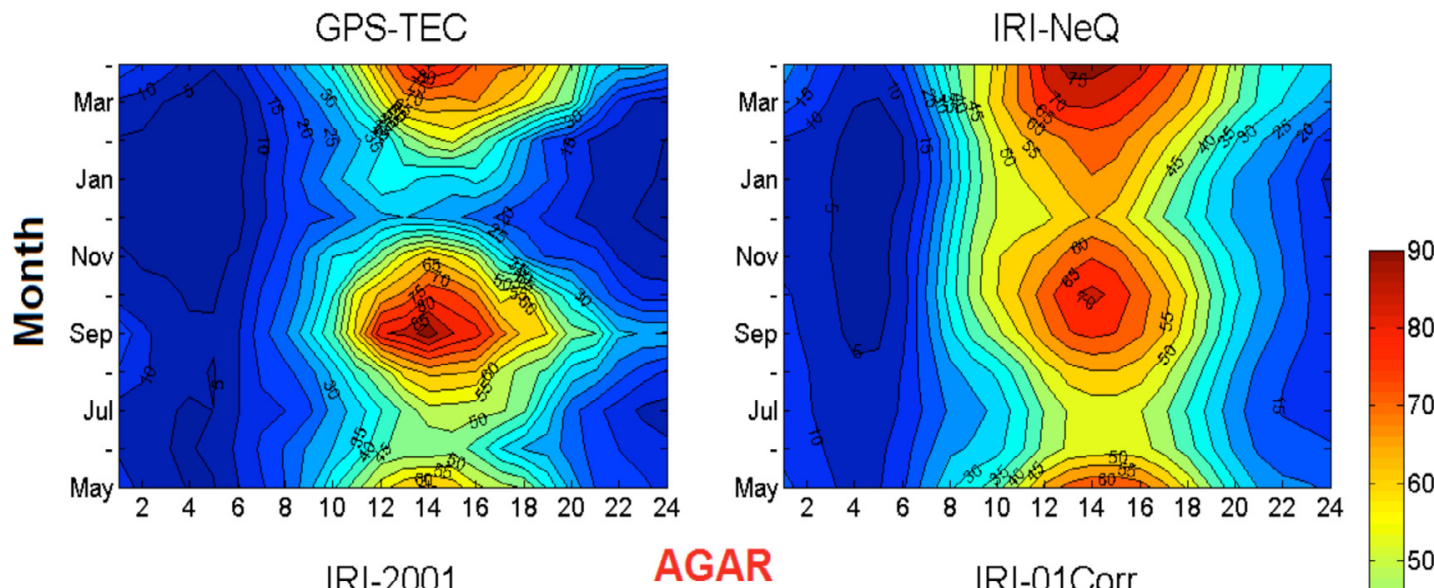

它

$|\mathrm{R}|-2001$
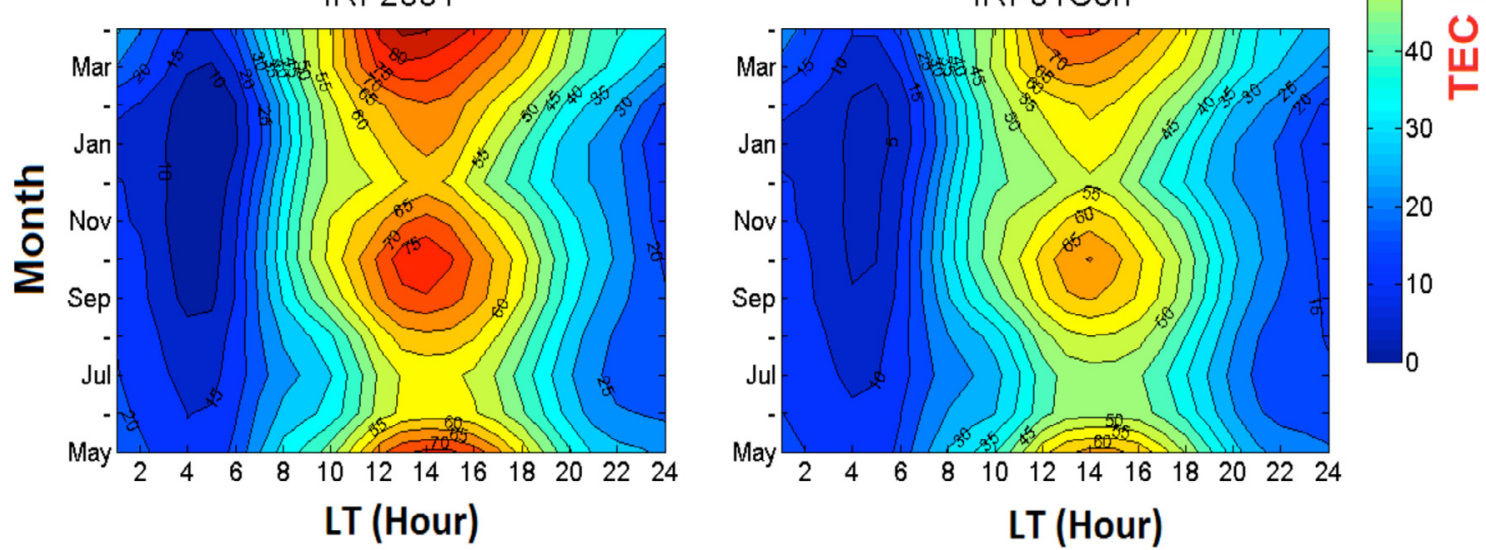

Figure 4. Contour diagram of monthly mean variation of GPS-TEC, IRI 2012 model TEC (NeQuick, IRI- 2001 and IRI-01-corr) at Agartala (AGAR) (Geomagnetic lat. $\left.14.22^{\circ} \mathrm{N}\right)$, from May 2012 to April 2013. 

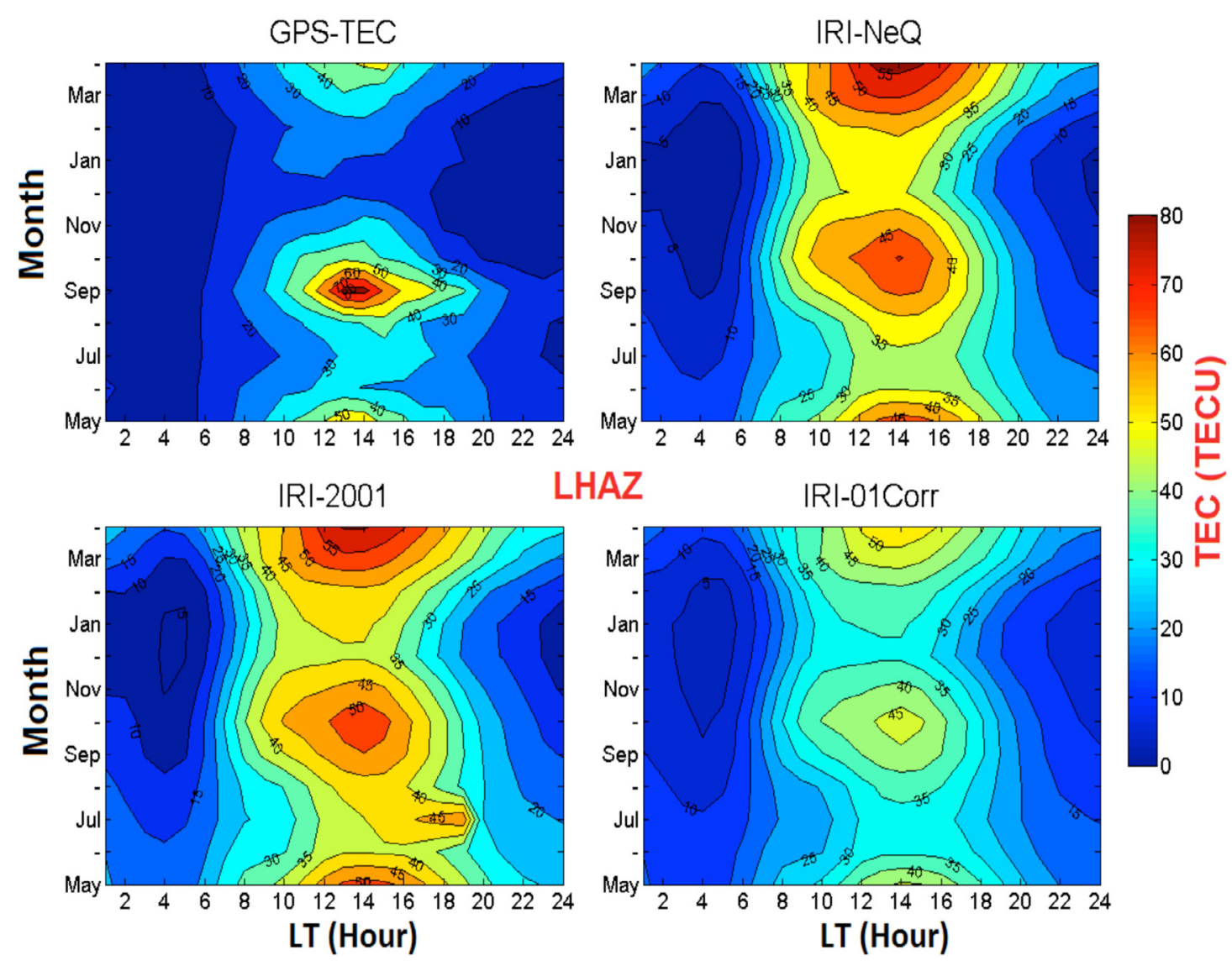

Figure 5. Contour diagram of monthly mean variation of GPS-TEC, IRI 2012 model TEC (NeQuick, IRI- 2001 and IRI-01-corr) at Lhasa (LHAZ) (Geomagnetic lat. $20.09^{\circ} \mathrm{N}$ ) from May 2012 to April 2013.
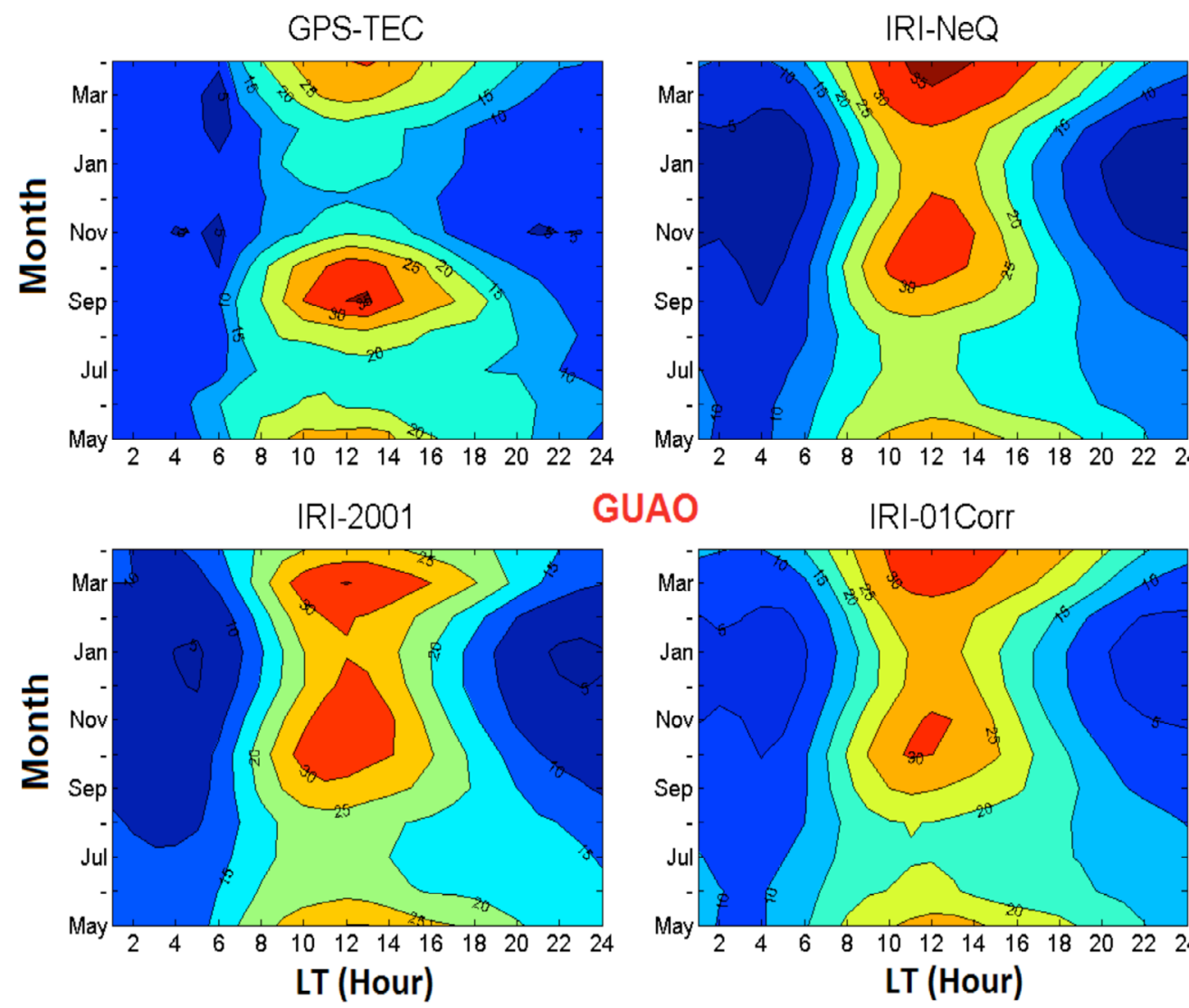

\section{GUAO IRI-01Corr}

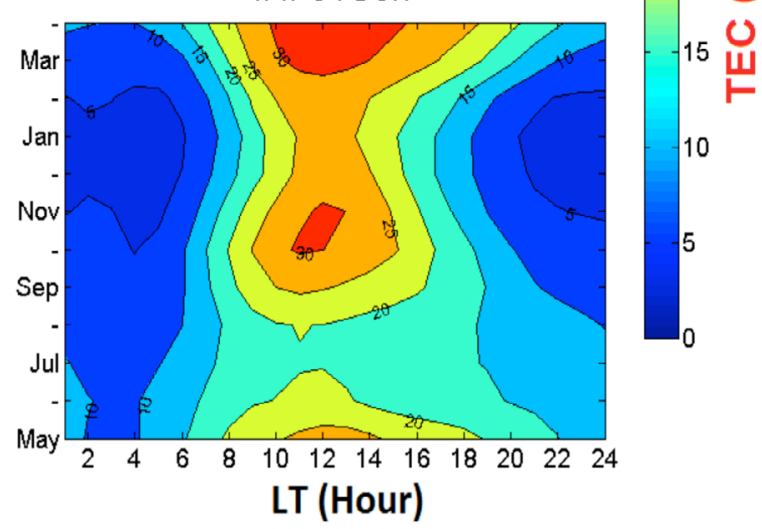

Figure 6. Contour diagram of monthly mean variation of GPS-TEC, IRI 2012 model TEC (NeQuick, IRI- 2001 and IRI-01-corr) at Urumqi (GUAO) (Geomagnetic lat. $34.04^{\circ} \mathrm{N}$ ) from May 2012 to April 2013. 
and October). The contour plots shown in Figures 3, 4, 5 and 6 respectively depict the monthly mean variations of TEC for all the months. It reflects that the maximum TEC has been observed for the equinox season from May 2012 to April 2013 for PBR2, AGAR, LHAZ and GUAO. The maximum TEC has been observed for AGAR station. About the seasonal variation of TEC, at all stations, the maximum TEC is observed in the equinox period while the lowest is observed in the winter.

In Figure 7, we have shown the seasonal mean TEC comparison of observed TEC and IRI 2012 TEC from May 2012 to April 2013 for all the stations. Figure 8 shows the difference in IRI model TEC as compared to GPS TEC (dTEC = IRI TEC - GPS TEC). During sum-
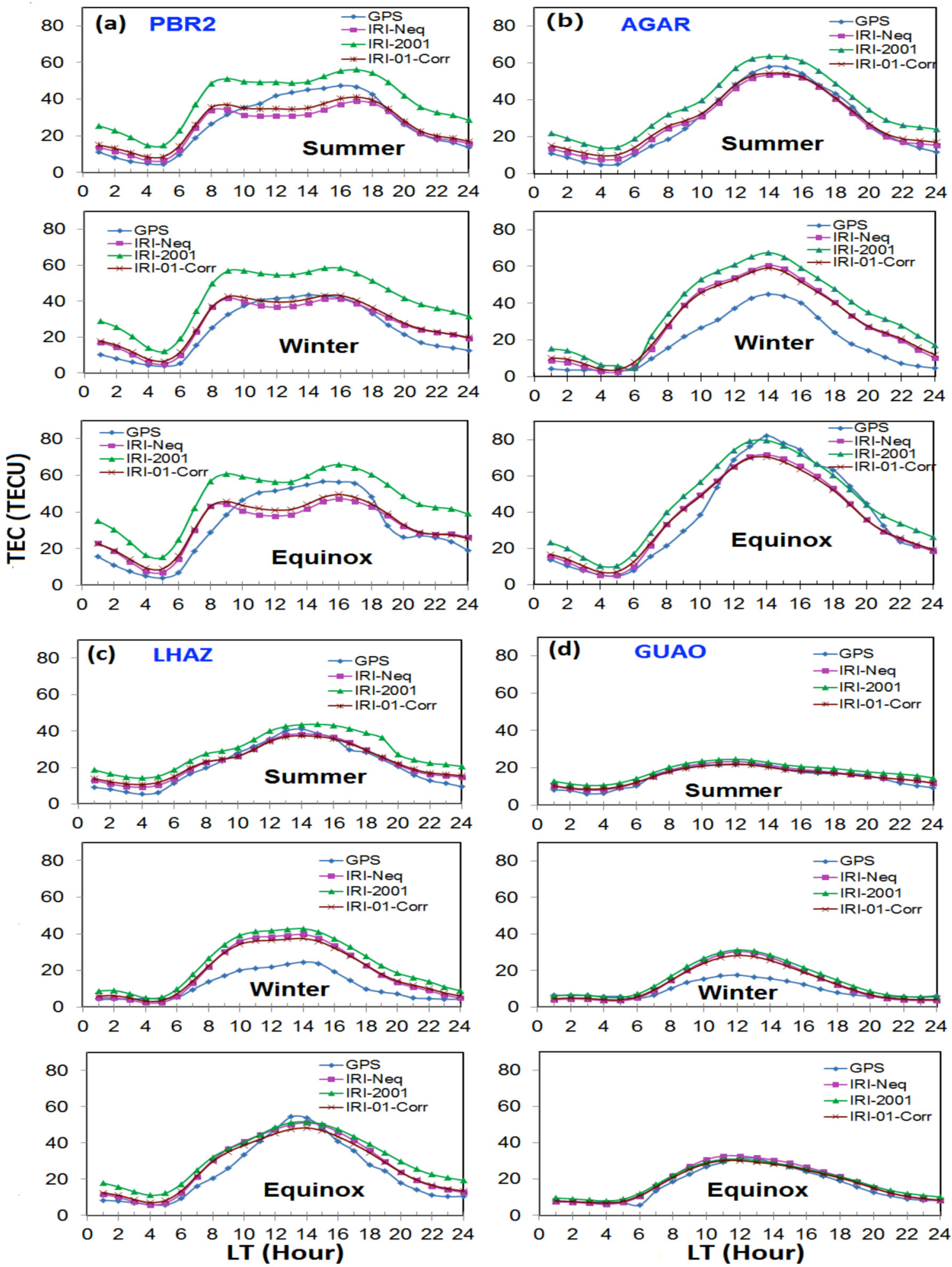

Figure 7. Seasonal variation of GPS-TEC and its comparison with IRI 2012 model TEC at (a) Port Blair (Geomagnetic lat. $2.11^{\circ}$ N) (b) Agartala (Geomagnetic lat. $\left.14.22^{\circ} \mathrm{N}\right)(\mathrm{c})$ Lhasa $\left(\right.$ Geomagnetic lat. $\left.20.09^{\circ} \mathrm{N}\right)$ and (d) Urumqi (Geomagnetic lat. $\left.34.04^{\circ} \mathrm{N}\right)$. 

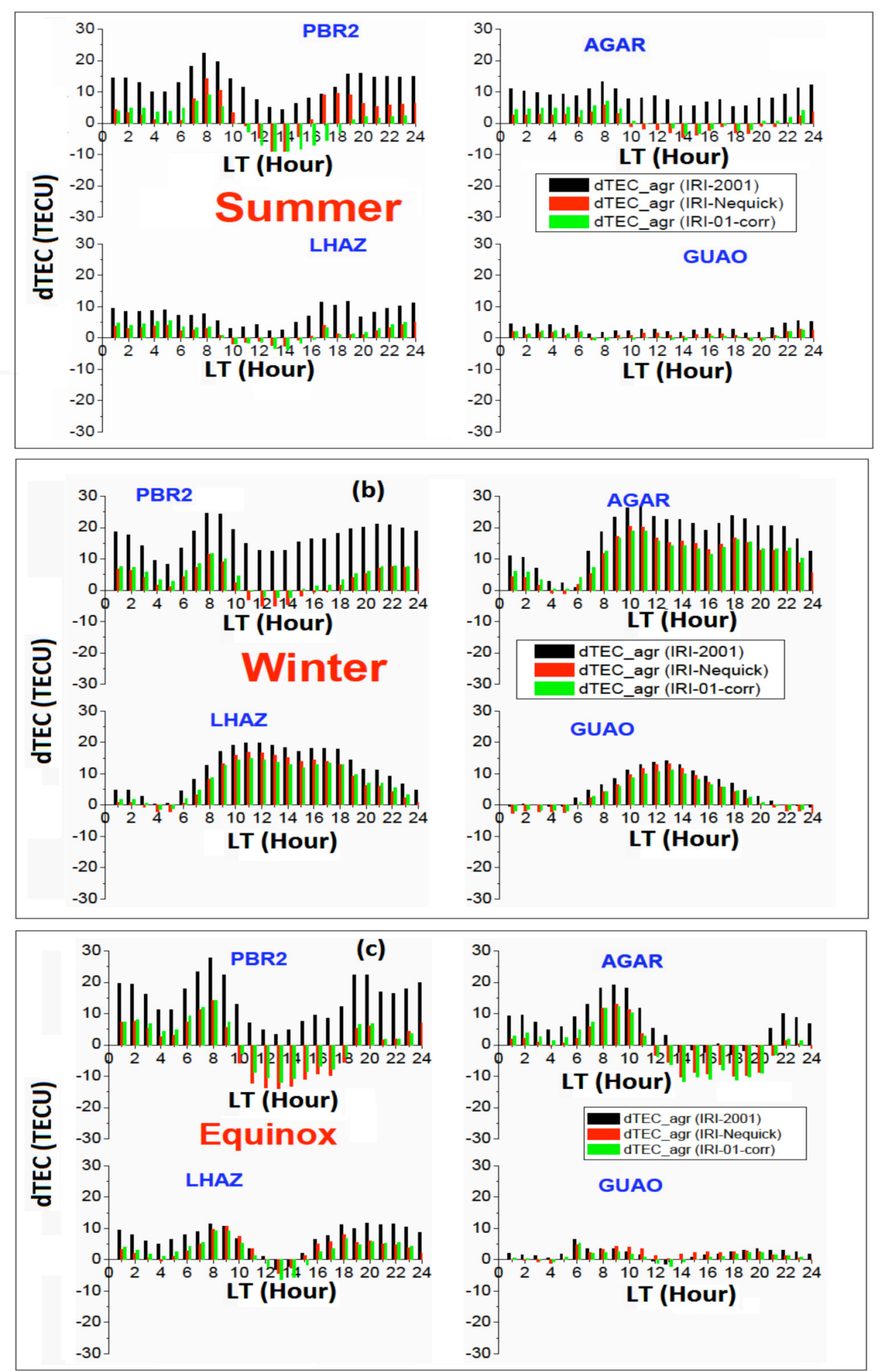

Figure 8. dTEC (IRI TEC - GPS TEC) variation for four stations during (a) summer (b) winter (c) Equinox.

mer time for all the four stations, the difference between the both TEC is minimum at around local noon times.

For PBR2, TEC from IRI-2001 option always overestimates the observed GPS TEC during all seasons. And this overestimation is highest in the winter period. During summer period at the noon time, from 9:30 to 17:30 LT, TEC from IRI-Nequick and IRI-01-corr option is underestimating the observed TEC. At the night time from
22:30 LT onwards, the IRI TEC is overestimating the observed TEC and this overestimation is remaining up to morning 8:30 LT. For winter season also during noon times, the IRI TEC from nequick and 01-corr option is underestimating observed TEC from 10:30 to 16:30 LT while in the night and morning time span, IRI is overestimating the observed TEC. During equinox season, the noon time 9:30 to 17:30 LT IRI TEC from NeQuick and 
01-corr option is underestimating the observed one and in the other times it is overestimating the observed one. Noon time difference between IRI and observed TEC is highest in the equinox, higher in the summer and less is the winter while the morning time observed TEC is always lower then IRI TEC. The difference between the IRI and nighttime TEC is maximum at the winter time. The noon bite out events is clearly as present in the IRI data but not observed in the GPS data.

In case of AGAR station, the IRI TEC always overestimates the observed TEC for winter season. For equinox season the IRI-2001 TEC shows a good agreement with the GPS TEC from 12:30 to 19:30 LT and outside this time span, it overestimates the observed TEC while during night time, the observed TEC matches with the TEC from NeQuick and IRI-01-corr options. In morning time, the IRI TEC from all the options overestimates the observed TEC. For summer season, the observed TEC agrees well with the TEC from NeQuick and IRI-01-corr options with around 34 TECU difference. IRI-2001 TEC overestimates the observed TEC for all the times of this season also. For Lasha station, IRI TEC from IRI-2001 option is always overestimating the observed TEC with the maximum difference in the winter season. TEC from IRI-Nequick and IRI-01-corr option underestimates the observed TEC from 11 to 17 LT. Except this time span, both the IRI options overestimate the observed TEC. During the night time after 2 to 8 LT, the IRI and the observed TEC are very close to each other. During equinox period, the noon peak is quite sharper than the summer and the winter seasons noon TEC of this station. During the noon period, the IRI TEC from all the three options is underestimating the observed TEC. At other times, the IRI TEC is overestimating the observed TEC. In case of GUAO station, the observed TEC closely matches with IRI TEC from 01-corr option up to 23 LT. Except this time, the IRI TEC is higher than the observed one. For winter season, the observed TEC is quite lower than the IRI TEC, although it closely matches with IRI2001 TEC from 24 to 8 LT. During equinox season, the observed TEC is lower then the IRI TEC up to 13 LT. After that period, up to $18 \mathrm{LT}$, both the TECs are almost equal and up to 1 LT, IRI overestimates the observed TEC. Again, there is a close match between the IRI and observed TEC after 2 LT. Table 1 depicts the correlation coefficients between the observed and model TEC. From Table 1, it is clear that matching is good between the IRI and GPS TEC in the low latitude and it is going to be better with increasing latitude.

\section{Discussion}

Our study shows that in general, the TEC predictions from IRI-2001 top side electron density option using IRI 2012 model overestimates the observed TEC. Similar observation for IRI-2001 was reported by several workers by using IRI 2007 model. It can also be noted from this study that as one move towards the higher latitudes, the IRI TEC from IRI-2001 option becomes good in agreement with the observed TEC. Rather than this the IRI- NeQuick and IRI-01-corr options TEC are close to each other. TEC from both these two options had a tendency to underestimate the observed TEC during the day time particularly in low latitude regions in the high solar activity period. These types of observations are quite consistent with the works of the previous workers [Coisson et al. 2008, Aggarwal 2011, Venkatesh et al. 2011, Kumar et al. 2012]. As noted earlier an underestimation is expected because the IRI-TEC does not include plasma sphere TEC. Therefore the IRI-TEC is expected to be lower than the GPS-TEC. Since the percentage contribution by the plasma sphere to GPS-TEC is much larger during the nighttime hour than the daytime hour. Therefore IRI model is expected to underestimate the GPS-TEC significantly during the nighttime than the daytime [Yizengaw et al. 2007]. In present study the IRI-TEC tends to underestimate the observed GPS-TEC in the day time hour (1200-1800 LT) during equinox (PBR2 and AGAR) season and summer season (PBR2) only particularly in the low-latitude region .

The large discrepancies (over estimation by IRI model) between GPS and IRI 2012 model TEC during all the time with maximum in the daytime hour have been observed in low latitude regions (PBR2, AGAR and LHAZ). One possible cause for the daytime dis-

\begin{tabular}{cccc}
\hline Stations & $\begin{array}{c}\text { Observed and IRI } \\
-2001 \text { TEC }\end{array}$ & $\begin{array}{c}\text { Observed and IRI } \\
\text {-01-corr TEC }\end{array}$ & $\begin{array}{c}\text { Observed and IRI } \\
\text {-NeQuick TEC }\end{array}$ \\
\hline PBR2 & 0.95394 & 0.95689 & 0.94027 \\
AGAR & 0.97229 & 0.97503 & 0.97554 \\
LHAZ & 0.98038 & 0.99635 & 0.98954 \\
GUAO & 0.99495 & 0.99627 \\
\hline
\end{tabular}

Table 1. List of correlation coefficient between the annual mean of observed and IRI TEC. 
crepancy (overestimation) in the IRI-2012 model may be the ionospheric expansion in low latitude regions to cover a larger slab thickness at around noon times in comparison to other times [Kenpankho et al. 2011]. This expansion at low latitude is larger as compared to mid latitude. At the equatorial regions, the steepest gradients, sharp peaks and deep valleys, and density crests are on both sides of the equator (due to Fountain effect), as explained by Bilitza and Reinisch [2008]. The occurrence of a noticeable trough supplemented by maximum TEC values in the pre-noon and the afternoon local time at equatorial regions is referred to as a noon bite-out, which is a characteristic feature at an equatorial station that falls in the trough of the equatorial ionization anomaly [Appleton 1946, Martyn 1955, Rastogi 1959]. Martyn [1955] and Rao [1966] showed that the pre-noon peak in the diurnal variation at equatorial latitudes is influenced by horizontal winds in addition to production and loss processes, while the afternoon peak is determined by vertical $\mathbf{E} \times \mathbf{B}$ electro dynamical drifts and diffusion along the magnetic field lines. Since the noon bite out is active only during the short period and therefore could not entirely explain the discrepancy in the IRI model as observed for the longer period in this study. This indicates that the daytime discrepancy (overestimation) in the IRI model, observed for a longer period (during all the time) in this study, is governed by some other parameters also.

The TEC from IRI model is not directly estimated from the measured TEC database, but the TEC calculation is conducted from foF 2 . The height profile of electron density is calculated based on the foF2, stepby-step. The density profile is then integrated along the vertical line to obtain TEC. In this process, topside electron density may not be satisfactorily modeled, i.e., the thickness is too small for IRI topside density profile. . The other possibility for the overestimation by the IRI model as observed during all time is the inaccurate prediction of foF 2 by the IRI model [Blitza et al. 2012]. The IRI bias during these time also could be due to overestimation of foF 2 by the CCIR coefficients. Further it can be noted that the topside thickness (i.e. topside scale height) in IRI is most inaccurate during the daytime period, but from Figure $9(a, b)$, it seems more likely that foF2 is inaccurate during this period.

During winter time, the IRI TEC always overestimates the observed TEC for AGAR, LHAZ and GUAO station. Same type of overestimation has also been found by Zhang et al. [2006]. To study the prediction accuracy of IRI 2012 model at F2 peak altitude (NmF2 and $\mathrm{hmF} 2$ ), we have taken the data of $\mathrm{NmF} 2$ and hmF2 from ionosonde measurement at Ahmedabad (Geog. Lat. $23.0^{\circ} \mathrm{N}$, long. $72.5^{\circ} \mathrm{E}$ ), India. The data at this station is available only for June, July and December
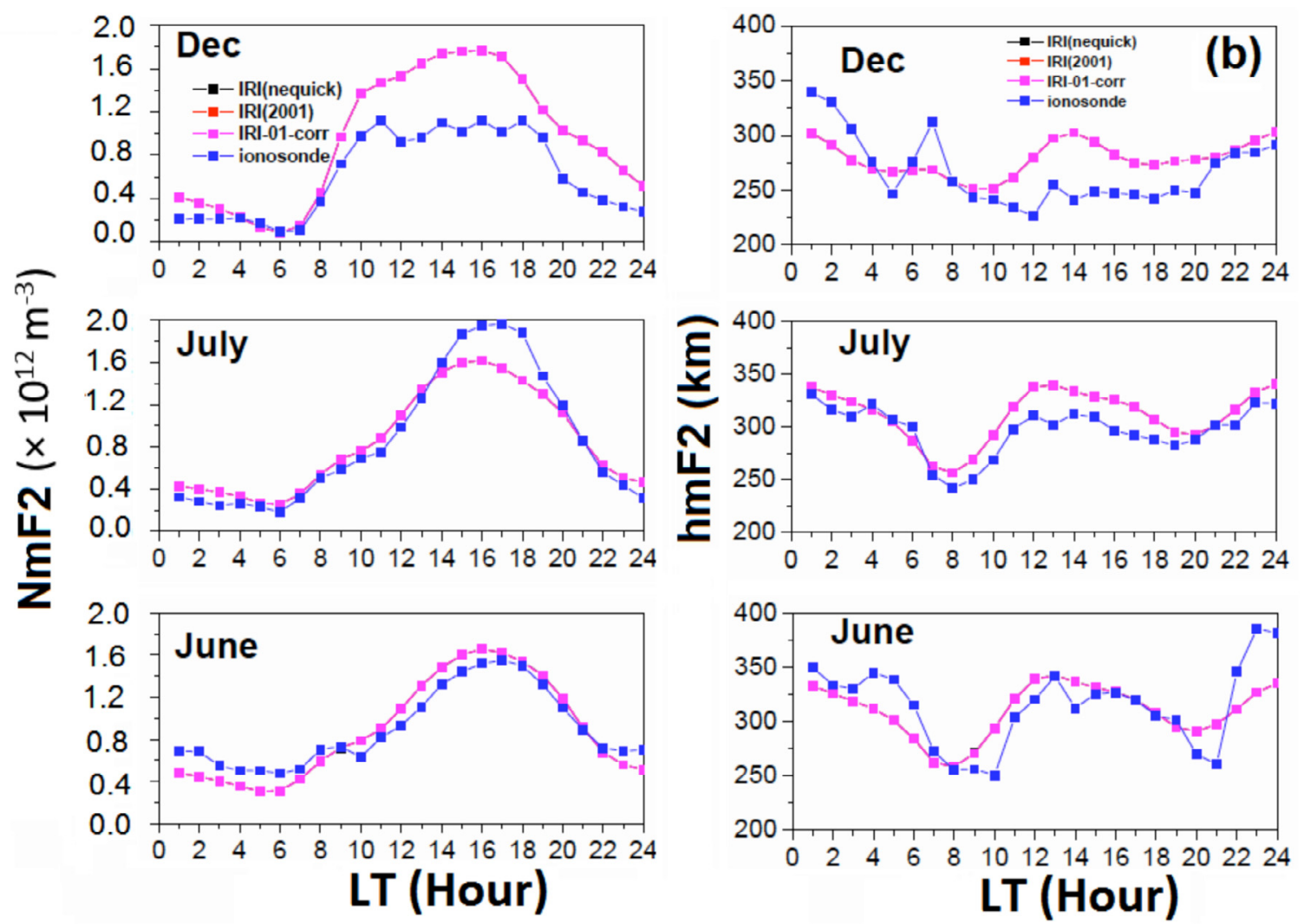

Figure 9. Comparison of IONOSONDE derived F2 peak electron density (NmF2) and height (hmF2) with IRI 2012 model during June, July and August of year 2012 over Ahmedabad, India. 
months of 2012. For these three months, the comparison of the hmF2 and NmF2 data of ionosonde with those obtained from IRI 2012 model and shown in Figure $9(a, b)$. From this figure, it is observed that there is a clear overestimation of ionosonde data at F2 peak (NmF2 and hmF2) by IRI model data during most of the hour in December month. However for the month of July, IRI hmF2 data is overestimating slightly and $\mathrm{NmF} 2$ data is underestimating the ionosonde data. In June, the data are closer to each other. So it can be seen that the inaccuracy in calculating the $\mathrm{NmF} 2$ as well as hmF2 data in December month could be the possible reason for overestimation of GPS-TEC data in winter season by IRI model data. Rather than this, in Figure 9, it can be noted that the $\mathrm{NmF} 2$ and hmF2 are not affected by the IRI topside options, so the lines corresponding to different topside options have been plotted on top of each other. The comparison shows that the CCIR coefficients contain significant errors that will overwhelm the topside contributions to TEC.

For LHAZ station, the rate of morning rise and afternoon decay in observed TEC is higher in the equinox season. This type of sharp gradient is absent in the IRI TEC for this season. In case of mid latitude station GUAO, during equinox, IRI TEC overestimates the observed TEC in the pre-noon and pre-afternoon time. During noon time period, IRI TEC is underestimating the observed TEC. During high solar activity period this type of observation has also found by Mosert et al. [2007] for a mid latitude station. It has also been observed that the IRI TEC from IRI-2001 topside electron density option overestimates the observed TEC in all time at low latitude. As one move towards the higher latitudes, the IRI TEC from IRI-2001 option becomes good in agreement with the observed TEC.

The difference between observation and IRI model depends on local time, latitude and strength of solar activity. Shastri et al. [1996] studied performance of IRI model using foF2 data observed from ionosonde and reported significant differences between observation and IRI model. The difference between observations and IRI model could also be attributed to longitude dependent shifts in the latitudinal position of the EIA towards higher the latitudes that occurs with increasing solar activity [Lyon and Thomas 1963]. This property is not satisfactory included in global prediction of IRI model

\section{Concluding remarks}

The present analysis shows that the TEC with topside option IRI-2001 overestimates the observed GPS TEC in low latitude regions in most of the times. The modeled TEC from other two options of IRI are in agreement with the observed TEC data. During high solar activity period of cycle 24 , the IRI TEC from the IRI-Nequick and IRI-01-corr options underestimates the observed TEC during noon time and overestimates during night time. So we can conclude from the above mentioned discussions that the matching between the IRI TEC and observed TEC is totally dependent on the local time, location and phase of the solar cycle. The largest deviations in model and observed TEC occur as a result of poor estimation of foF 2 and $\mathrm{NmF} 2$ from the coefficients of IRI model. These results might be useful for the model improvement and the model error representation of the data assimilation. The error in IRI model TEC with respect to ground based GPS measurements at low latitude regions is found to be higher than mid-latitude during all the seasons.

Acknowledgements. The authors are thankful to International GNSS Service (IGS) team for providing the IGS stations data (ftp://igscb.jpl.nasa.gov/). For bias calculation DCB files has been taken from ftp:/ / ftp.unibe.ch/aiub/CODE/ website. The IRI 2012 model data has been downloaded from the web interface (http:/ / www.omniweb.gsfc.nasa.gov/vitmo/iri_vitmo.html). Authors also thankful to Geoscience Australia (http:/ / www.ga.gov.au/oracle/ geomag/iqd_form.jsp) for providing the International quiet days. Ionosonde data has been taken from the website http: / / ulcar.uml. edu/DIDBase/. The authors also wish to express their cordial thank to the anonymous reviewer for his constructive suggestions which helped us to improve the manuscript.

\section{References}

Aggarwal., M. (2011). TEC variability near northern EIA crest and comparison with IRI model, Adv. Space Res., 48 (7), 1221-1231.

Anderson, D.N., M. Mendillo and B. Herniter (1987). A semi-empirical low latitude ionospheric model, Radio Sci., 22 (2), 292-306.

Appleton, E.V. (1946). Two anomalies in ionosphere, Nature, 157, 691-693.

Azzarone, A., C. Bianchi, M. Pezzopane, M. Pietrella, C. Scotto and A. Settimi (2012). IONORT: A Windows software tool to calculate the HF ray tracing in the ionosphere, Comp. Geosc., 42, 57-63.

Bagiya, M.S., H.P. Joshi, K.N. Iyer, M. Aggarwal, S. Ravindran and B.M. Pathan (2009). TEC variations during low solar activity period (2005-2007) near the Equatorial Ionospheric Anomaly Crest region in India, Annales Geophysicae, 27 (3), 1047-1057.

Balan, N., P.B. Rao and K.N. Iyer (1986). Seasonal and solar variations of night-time anomalous enhancements in total electron content, Proc. Indian Acad. Sci., 95, 409-416.

Balan, N., G.J. Bailey and R. Balachandran Nair (1991). Solar and mag- netic activity effects on the latitudinal variations of night-time TEC enhancement, Annales Geophysicae, 9, 60-69. 
Balan, N., Y. Otsuka and S. Fukao (1997). New aspects in the annual variation of the ionosphere observed by the MU Radar, Geophys. Res. Lett., 24, 2287-2290.

Bhuyan, P.K., and R.R. Borah (2007). TEC derived from GPS network in India and comparison with the IRI, Adv. Space Res., 39 (5), 830-840.

Bilitza, D. (1986). International reference ionosphere: recent developments, Radio Sci., 21 (3), 343-346.

Bilitza, D. (1990). International reference ionosphere 1990, NSSDC/WDC-A-R\&S 90-22, National Space Science Data Center, Greenbelt.

Bilitza, D. (2001). International reference ionosphere 2000, Radio Sci., 36 (2), 261-275.

Bilitza, D., and B.W. Reinisch (2008). International reference ionosphere 2007 improvements and new parameters, Adv. Space Res., 42 (4), 599-609.

Bilitza, D., S.A. Brown, Y.W. Mathew, R.S. Jonas and A.R. Patrick (2012) Measurements and IRI model predictions during the recent solar minimum, J Atmos. Sol.-Terr. Phy., 86, 99-106.

Booker, H.G. (1977). Fitting of multi region ionospheric profiles of electron density by a single analytic function of height, J. Atmos. Sol.-Terr. Phys., 39 (5), 619-623.

Bramley, E.N., and M. Young (1968). Winds and electromagnetic drifts in the equatorial F2-region, J. Atmos. Terr. Phys., 30, 99-111.

CCIR, Comité Consultatif International des Radiocommunications (1966). Report 340-1 and 340-6, International Telecommunication Union, Geneva, Switzerland.

Chauhan,V., and O.P. Singh (2010). A morphological study of GPS-TEC data at Agra and their comparison with the IRI model, Adv. Space Res., 46, 280-290.

Coisson, P., S.M. Radicella, L. Ciralo, R. Leitinger and B. Nava (2008). Global validation of IRI TEC for high and medium solar activity conditions, Adv. Space Res., 42, 770-775.

Daniell, R.E., and L.D. Brown (1995). PRISM, A Parameterized Real-Time Ionospheric Specification Model Version 1.5, Computational Physics Inc., Newton.

da Rosa, A.V., H. Waldman, J. Bendito and O.K. Garriott (1973). Response of the ionospheric electron content to fluctuations in solar activity, J. Atmos. Terr. Phys., 35, 1429-1442.

Ezquer, R.G., C.A. Jadur and M. Mosert de Gonzalez (1998). IRI-95 TEC predictions for the South American peak of the equatorial anomaly, Adv. Space Res., 22 (6), 811-814.

Hofmann-Wellenhof, B., H. Lichtenegger and J. Collins (2001). Global Positioning System: Theory and Practice, Springer, Berlin.

Jain, A., S. Tiwari, S. Jain and A.K. Gawl (2011). Night- time enhancements in TEC near the crest of northern equatorial ionization anomaly during low solar activity period, Indian J. Phys., 85 (9), 1367-1380.

Johnson, F.S. (1963). Composition changes in the upper atmosphere, In: E. Thrane (ed.), Electron Density Distributions in the Ionosphere and Exosphere, North-Holland, Amsterdam, 81-84.

Kenpankho, P., K. Watthanasangmechai, P. Supnithi, T. Tsugawa and T. Maruyama (2011). Comparison of GPS TEC measurements with IRI TEC prediction at the equatorial latitude station, Chumphon, Thailand, Earth Planets Space, 63, 365-370.

Klobuchar, J. (1986). Design and characteristics of the GPS ionospheric time-delay algorithm for single-frequency users, In: Procedings of the IEEE Position Location and Navigation Symposium (Las Vegas, November 4-7).

Kumar, S., and A.K. Singh (2009). Variation of ionospheric total electron content in Indian low latitude region of equatorial ionization anomaly (EIA), J. Adv. Space Res., 43, 1555-1562.

Kumar, S., S. Priyadarshi, S. Gopi Krishna and A.K. Singh (2012). GPS-TEC variations during low solar activity period (2007-2009) at Indian low latitude stations, Astrophys. Space Sci., 339 (1), 165-178.

Lanzerotti, L.J., L.L. Cogger and M. Mendillo (1975). Latitude dependence of ionosphere total electron content: Observations during sudden commencement storms, J. Geophys. Res., 80, 1287-1306.

Liu, Z., Y. Gao and S. Skone (2005). A study of smoothed TEC precision inferred from GPS measurements, Earth Planets Space, 57, 999-1007.

Liu, L., W. Wan, M.-L. Zhang and B. Zhao (2008). Case study on total electron content enhancements at low latitudes during low geomagnetic activities before the storms, Annales Geophysicae, 26, 893-903.

Lyon, A.J., and L. Thomas (1963). The F2-region equatorial anomaly in the African, American and East Asian sectors during sunspot maximum, J. Atmos. Terr. Phys., 25, 373-386.

Maltseva, O.A., N.S. Mozhaeva, O.S. Poltavsky and G.A. Zhbankov (2012). Use of TEC global maps and the IRI model to study ionospheric response to geomagnetic disturbances, Adv. Space Res., 49 (6), 10761087.

Martyn, D.F. (1955). Theory of height and ionization density changes at the maximum of a chmpman-like region, taking account of ion production, decay, diffusion and total drift, Proceedings Cambridge Conference, Physical Society, London, 254-259.

Mosert, M., M. Gende, C. Brunini, R.G. Ezquer and D. Altadill (2007). Comparisons of IRI TEC predictions with GPS and digisonde measurements at Ebro, 
Adv. Space Res., 39, 841-847.

Mukherjee, S., S. Sarkar, P.K. Purohit and A.K. Gwal (2010). Seasonal variation of total electron content at crest of equatorial anomaly station during low solar activity conditions, Adv. Space Res., 46 (3), 291-295.

Nava, B., P. Coisson and S.M. Radicella (2008). A new version of the NeQuick ionosphere electron density model, J. Atmos. Sol.-Terr. Phys., 70 (15), 1856-1862.

Rama Rao, P.V.S., S. Gopi Krishna, K. Niranjan and D.S.V.V.D. Prasad (2006a). Temporal and spatial variations in TEC using simultaneous measurements from the Indian GPS network of receivers during the low solar activity period of 2004-2005, Annales Geophysicae, 24, 3279-3292.

Rama Rao, P.V.S., K. Niranjan, D.S.V.V.D. Prasad, S. Gopi Krishna and G. Uma (2006b). On the validity of the ionospheric pierce point (IPP) altitude of 350 $\mathrm{km}$ in the Indian equatorial and low-latitude sector, Annales Geophysicae, 24, 2159-2168.

Rao, B.C.N. (1966). Control of equatorial Spread-F by the F layer height, J. Atmos. Terr. Phys., 28, 1207-1217.

Rao, G.S. (2007). GPS satellite and receiver instrumental biasesestimation using least squares methodfor accurate ionosphere modelling, J. Earth Syst. Sci., 116 ( 5), 407-411.

Rastogi, R.G. (1959). The diurnal development of the anomalous equatorial belt in the $\mathrm{F} 2$ region of the ionosphere, J. Geophys. Res., 64, 727-732.

Rastogi, R.G., and R.P. Sharma (1971). Ionospheric electron content at Ahmedabad (near the crest of equatorial anomaly) by using bea-con satellite transmission during half a solar cycle, Planet. Space Sci., 19, 15051517.

Rawer, K., D. Bilitza and S. Ramakrishnan (1978). Goals and status of international reference ionosphere, Rev. Geophys., 16 (2), 177-181.

Rishbeth, H., and C.S.G.K. Setty (1961). The F-layer at sunrise, J. Atmos. Terr. Phys., 20, 263.

Rush, C., M. Fox, D. Bilitza, K. Davies, L. Mcnamara, F. Stewart and M. Pokempner (1989). Ionospheric mapping - an update of foF 2 coefficients, Telecomm. J., 56, 179-182.

Sardon, E., A. Rius and N. Zarraoa (1994). Estimation of the transmitter and receiver differential biases and the ionospheric total electron content from Global Positioning System observations, Radio Sci., 29 (3), 577- 586.

Settimi, A., M. Pezzopane, M. Pietrella, C. Bianchi, C. Scotto, E. Zuccheretti and J. Makris (2013). Testing the IONORT-ISP system: A comparison between synthesized and measured oblique ionograms, Radio. Sci., 48, 167-179.

Shastri, S., S. Aggarwal and N.K. Sethi (1996). Perform- ance of IRI model prediction of F-region for Indian latitude, Adv. Space Res., 18 (6), 41-44.

Sojka, J., M. David, R.W. Schunk and R.A. Heelis (2012). A modeling study of the longitudinal dependence of storm time midlatitude dayside total electron content enhancements, J. Geophys. Res., 117, A02315.

Sridharan, R., D. Pallam Raju and R. Raghavarao (1994). Precursor to equatorial spread-F in OI $630.0 \mathrm{~nm}$ day glow, Geophys. Res. Lett., 21 (25), 2797-2800.

Stening, R.J. (1992). Review paper modeling the low latitude F-region, J. Atmos. Terr. Phys., 54, 1387-1421.

Torr, M.R., and D.G. Torr (1973). The seasonal behaviour of the F2-layer of the ionosphere, J. Atmos. Terr. Phys., 35, 2237-2251.

Venkatesh, K., P.V.S. Rama Rao, P.L. Saranya, D.S.V.V.D. Prasad and K. Niranjan (2011). Vertical electron density and topside effective scale height (HT) variations over the Indian equatorial and low latitude stations, Annales Geophysicae, 29, 1861-1872.

Warnant, R. (2000). The increase of ionospheric activity as measured by GPS, Earth Planets Space, 52, 1055-1060.

Wu, C.C., C.D. Fry, K. Liou and C.L. Tseng (2004). Annual TEC variation in the equatorial anomaly region during the solar minimum: September 1996 -August 1997, J. Atmos. Terr. Phys., 66, 199-207.

Wu, C.C., C.D. Fry, K. Liou, S.-J. Shan and C.L. Tseng (2008). Variation of ionospheric total electron content in Taiwan region of the equatorial anomaly from 1994 to 2003, Adv. Space Res., 41, 611-616.

Yizengaw, E., M.B. Moldwin, P.L. Dyson and E.A. Essex (2007). Using tomography of GPS TEC to routinely determine ionospheric average electron density profiles, J. Atmos. Sol.-Terr. Phys., 69 (3), 314-321.

Zhang, M.L., S.M. Radicella, J.K. Shi, X. Wang and S.Z. $\mathrm{Wu}$ (2006). Comparison among IRI, GPS-IGS and ionogram-derived total electron contents, Adv. Space Res., 37, 972-977.

Zou, S., M.B. Moldwin, A. Coster, L.R. Lyons and M.J. Nicolls (2011). GPS TEC observations of dynamics of the mid latitude trough during substorms, Geophys. Res. Lett., 38, L14109.

\footnotetext{
${ }^{\star}$ Corresponding author: Anirban Guha,

Tripura University, Department of Physics, Suryamaninagar,

Tripura, India; email: anirban1001@yahoo.com.

C 2014 by the Istituto Nazionale di Geofisica e Vulcanologia. All rights reserved.
} 